\title{
Author Correction: Metrological complementarity reveals the Einstein-Podolsky-Rosen paradox
}

\author{
Benjamin Yadin, Matteo Fadel (D) \& Manuel Gessner (D)
}

Correction to: Nature Communications https://doi.org/10.1038/s41467-021-22353-3, published online 23 April 2021.

The original version of this Article contained errors in inline Equations in the last paragraph of the "Steering of GHZ states" subsection, which incorrectly read:

For a mixture $\rho=p\left|\mathrm{GHZ}_{\phi}{ }^{N+1}\right\rangle\left\langle\mathrm{GHZ}_{\phi}{ }^{N+1}\right|+(1-p) 1 / 2^{N+1}$, using the same measurements we obtain $F_{\mathrm{Q}}{ }^{\mathrm{B} \mid \mathrm{A}}\left[\rho, J_{z}\right] \geq p^{2} N^{2} /[p+$ $\left.2(1-p) / 2^{N}\right], 4 \operatorname{Var}_{\mathrm{Q}}{ }^{\mathrm{B} \mid \mathrm{A}}[\rho, J z] \leq(1-p) N / 2^{N}+p(1-p) N^{2}$. Whenever $p \gg 2^{-N}$, the criterion witnesses steering.

The correct form should read:

For a mixture $\rho=p\left|\mathrm{GHZ}_{\phi}{ }^{N+1}\right\rangle\left\langle\mathrm{GHZ}_{\phi}{ }^{N+1}\right|+(1-p) 1 / 2^{N+1}$, using the same measurements we obtain $F_{\mathrm{Q}} \mathrm{B}^{\mathrm{B}}\left[\rho, J_{z}\right] \geq p^{2} N^{2} /[p+$ $\left.2(1-p) / 2^{N}\right], 4 \operatorname{Var}{ }_{Q} B \mid A[\rho, J z] \leq(1-p) N+p(1-p) N^{2}$. For large $N$, whenever $p \gtrsim 1 / \sqrt{ } N$, the criterion witnesses steering.

This has been corrected in the PDF and HTML versions of the Article.

The original version of the Supplementary Information associated with this Article contained errors in the last paragraph of Supplementary Note 2 "GHZ States with white noise", which incorrectly read:

For a measurement of $\sigma_{z}$ by Alice, Bob's conditional states are easily found to give

$$
\operatorname{Var}_{\mathrm{Q}}^{\mathrm{B} \mid \mathrm{A}}\left[\rho, J_{z}\right] \leq \frac{(1-p) N}{4 d}+\frac{p(1-p) N^{2}}{4}
$$

where $d=2^{N}$. With a $\sigma_{x}$ measurement, (3) results in

$$
F_{\mathrm{Q}}^{\mathrm{B} \mid \mathrm{A}}\left[\rho, J_{z}\right] \geq \frac{p^{2} N^{2}}{p+2(1-p) / d} .
$$

When $p \gg 1 / d=2^{-N}$, we can neglect the terms involving $d$. Then $F_{\mathrm{Q}}{ }^{\mathrm{B}}\left|\mathrm{A}\left[\rho, J_{z}\right] \gtrsim p N^{2}, \operatorname{Var}_{\mathrm{Q}}{ }^{\mathrm{B}}\right| \mathrm{A}\left[\rho, J_{z}\right] \lesssim p(1-p) N^{2}<p N^{2}$ for $p<1$.

The correct version reads instead

For a measurement of $\sigma_{z}$ by Alice, Bob's conditional states are easily found to give

$$
\operatorname{Var}_{\mathrm{Q}}^{\mathrm{B} \mid \mathrm{A}}\left[\rho, J_{z}\right] \leq \frac{(1-p) N}{4}+\frac{p(1-p) N^{2}}{4},
$$

With a $\sigma_{x}$ measurement, (3) results in

$$
F_{\mathrm{Q}}^{\mathrm{B} \mid \mathrm{A}}\left[\rho, J_{z}\right] \geq \frac{p^{2} N^{2}}{p+2(1-p) / d},
$$

where $d=2^{N}$. When $p \gg 1 / d=2^{-N}$, we can neglect the term involving $d$. Then $F_{\mathrm{Q}}{ }^{\mathrm{B} \mid \mathrm{A}}\left[\rho, J_{z}\right] \gtrsim p N^{2}$, and the difference

$$
F_{\mathrm{Q}}^{\mathrm{B} \mid \mathrm{A}}\left[\rho, J_{z}\right]-4 \operatorname{Var}_{\mathrm{Q}}^{\mathrm{B} \mid \mathrm{A}}\left[\rho, J_{z}\right] \gtrsim p^{2} N^{2}-(1-p) N
$$

is positive as long as $N>(1-p) / p^{2}$. For large $N$, this condition approximates to $p \gtrsim 1 / \sqrt{ } N$. 
The HTML has been updated to include a corrected version of the Supplementary Information.

Published online: 04 November 2021

\section{Additional information}

Supplementary information The online version contains supplementary material available at https://doi.org/10.1038/s41467-021-26597-x.

(c) Open Access This article is licensed under a Creative Commons Attribution 4.0 International License, which permits use, sharing, adaptation, distribution and reproduction in any medium or format, as long as you give appropriate credit to the original author(s) and the source, provide a link to the Creative Commons license, and indicate if changes were made. The images or other third party material in this article are included in the article's Creative Commons license, unless indicated otherwise in a credit line to the material. If material is not included in the article's Creative Commons license and your intended use is not permitted by statutory regulation or exceeds the permitted use, you will need to obtain permission directly from the copyright holder. To view a copy of this license, visit http://creativecommons.org/licenses/by/4.0/.

(C) The Author(s) 2021 